\title{
Thrombocytopenia and splenic platelet directed immune responses after intravenous ChAdOx1 nCov-19 administration.
}

Leo Nicolai ${ }^{1,2, \#, \S}$, Alexander Leunig ${ }^{1,2, \#, \S, ~ K a m i ~ P e k a y v a z}{ }^{1,2, \#, \S}$, Afra Anjum $^{1}$, Eva Riedlinger ${ }^{1}$, Luke Eivers ${ }^{1}$, Marie-Louise Hoffknecht ${ }^{1}$, Dario Rossaro ${ }^{1}$, Raphael Escaig ${ }^{1}$, Rainer Kaiser ${ }^{1,2}$, Vivien Polewka ${ }^{1}$, Anna Titova $^{1}$, Karsten Spiekermann ${ }^{3}$, Matteo lannacone ${ }^{4}$, Konstantin Stark ${ }^{1,2, *}, \S$, Steffen Massberg ${ }^{1,2, *}$

1 Medizinische Klinik und Poliklinik I University Hospital Ludwig-Maximilian University Munich, Germany 2 DZHK (German Centre for Cardiovascular Research), partner site Munich Heart Alliance, Germany 3 Medizinische Klinik und Poliklinik III University Hospital Ludwig-Maximilian University Munich, Germany 4 Division of Immunology, Transplantation and Infectious Diseases, IRCCS San Raffaele Scientific Institute, Milan, Italy.

\# Contributed equally

* Jointly supervised this work

$\S$ Corresponding authors

\section{Summary paragraph}

Vaccines against SARS-CoV-2 are based on a range of novel vaccine platforms, with adenovirus-based approaches (like ChAdOx1 nCov-19) being one of them. Recently a rare and novel complication of SARSCoV-2 targeted adenovirus vaccines has emerged: thrombosis with thrombocytopenia syndrome (TTS). TTS is characterized by low platelet counts, clot formation at unusual anatomic sites and plateletactivating PF4-polyanion antibodies reminiscent of heparin-induced thrombocytopenia. Here, we employ in vitro and in vivo models to characterize the possible mechanisms of this platelet-targeted autoimmunity. We show that intravenous but not intramuscular injection of ChAdOx1 nCov-19 triggers platelet-adenovirus aggregate formation and platelet activation. After intravenous injection, these aggregates are phagocytosed by macrophages in the spleen and platelet remnants are found in the marginal zone and follicles. This is followed by a pronounced B-cell response with the emergence of circulating antibodies binding to platelets. Our work contributes to the understanding of TTS and highlights accidental intravenous injection as potential mechanism for post-vaccination TTS. Hence, safe intramuscular injection, with aspiration prior to injection, could be a potential preventive measure when administering adenovirus-based vaccines.

\section{Main text}

The emergence of SARS-CoV-2 and the ensuing pandemic has led to the development of vaccines eliciting an immune response to the virus spike protein in an unprecedented time frame. Multiple vaccine platforms have been deployed successfully, with mRNA and adenovirus-vector based vaccines carrying the bulk of global vaccination efforts [1]. Recently, a potentially novel adverse effect of vaccination termed thrombosis with thrombocytopenia syndrome (TTS) or vaccine induced thrombotic thrombocytopenia (VITT) has been described in patients receiving adenovirus-based vaccines (ChAdOx1 nCov-19 
(AstraZeneca) and Ad26.COV2.S (Johnson\&Johnson) 4-30 days prior [2, 3]. This rare disease resembles heparin induced thrombocytopenia type II (HIT II) with positive PF4-polyanion antibody assay required for diagnosis $[4,5]$. However, the underlying mechanism of TTS development only in a fraction of vaccinated individuals remain poorly understood and potential preventive measures are not established[6].

A previously healthy 27-year-old male presented to our emergency department with new onset headache non-responsive to ibuprofen 10 days after vaccination with ChAdOx1 nCov-19 (Fig. 1a). Initial evaluation revealed thrombocytopenia and elevated D-Dimer, as well as a positive rapid HIT screening test (Suppl. Fig. 1a and Suppl. Table $1 \& 2$ ). A contrast enhanced head CT scan was concordant with sinus vein thrombosis (SVT), an otherwise uncommon site of thrombosis suggestive of TTS (Fig. 1b). The patient was treated with IVIGs and anticoagulated with argatroban. He was discharged upon disappearance of symptoms and anticoagulation was continued with dabigatran. Thrombophilia screening was inconclusive (Suppl. Table 1). Evaluation of risk factors revealed current smoking. Tests used for the detection/confirmation of relevant PF4/polyanion antibodies (HIT-ELISA, HIT-IL-ACUSTAR and HIPA/PIPA test) were all negative in this patient, formally ruling out TTS according to guidelines[5] (Suppl. Table 2). Consistent with the case described here, reports on thrombotic and thrombocytopenic complications after adenovirus-based SARS-CoV-2 vaccination include an increased rate of isolated thrombocytopenia, as well as PF4-polyanion negative cases of sinus vein thromboses $[7,8]$. We therefore asked if additional mechanisms beyond PF4/polyanion antibodies - potentially involving direct adenoviral vector-platelet interaction - might be involved in the pathogenesis of TTS.

To study direct adenoviral vector-platelet interaction, we incubated healthy human platelets with ChAdOx1 nCov-19, mRNA-based vaccine BNT162b2 (Pfizer-BioNTech) or phosphate buffered saline (PBS). Incubation with anti-pan-Adenovirus antibody revealed binding of ChAdOx1 nCov-19 to human platelets (Fig. 1c-d). This binding resulted in a shift of platelets towards an activated phenotype with increased activated GPIIb-IIla integrin (Fig. 1e, Suppl Fig. 1b). Similarly, mouse platelets also strongly bound ChAdOx1 nCov-19 in-vitro (Suppl Fig. 1c).

Vaccines are routinely administered intramuscularly (i.m.) and trigger immune responses mainly in the draining lymph nodes [9]. Based on our finding that adenoviral vaccine binds to blood platelets, we hypothesized that accidental intravenous injection of adenoviral vaccine might lead to platelet-adenovirus aggregate formation with platelet activation and possibly platelet clearance. Indeed, intravenous but not i.m. injection of ChAdOx1 nCov-19 triggered transient thrombocytopenia in mice (Fig. 1f). Intravenous injection of PBS, BNT162b2 or heat inactivated ChAdOx1 nCov-19 injection had no effect on platelet counts (Fig. 1f). Thrombocytopenia induced by intravenous injection of ChAdOx1 nCov-19 was not due to fluid shift or changes in platelet production, as indicators like platelet distribution width, mean platelet volume and platelet larger cell ratio (P-LCR) did not show variations (Suppl Fig. 1d). The decline in platelet count was dose-dependent and correlated directly with adenovirus-positive platelets circulating in the blood one hour after i.v. injection (Fig. 1g, Suppl Fig. 1e). AID ${ }^{-/}$slgM $^{-/-}$mice lacking IgM and IgG antibodies displayed the same platelet count kinetic, indicating that development of thrombocytopenia within $48 \mathrm{hr}$ after intravenous injection of ChAdOx1 nCov-19 does not depend on antibodies (Suppl. Fig. 1f). However, intravenous but not i.m. injection of ChAdOx1 nCov-19 resulted in a strong increase in platelet-adenovirus aggregates (Fig. 1h). These aggregates had a short dwell time and rapidly disappeared from the circulation (Fig. 1h). Disappearance of platelet-adenovirus aggregates preceded recovery of platelet counts (Fig $1 \mathrm{f}$ and $\mathrm{h}$ ). To further investigate if binding of adenovirus is responsible for enhanced platelet clearance, we co-injected labeled platelets incubated with either ChAdOx1 nCov-19 or BNT162b2. We observed that 
ChAdOx1 nCov-19 incubated platelets were selectively removed from the circulation (Fig.1i) (2.8 $\pm 1.1 \mathrm{~h}$ half-life for ChAdOx1 nCov-19 incubated platelets vs. 10 $\pm 3.7 \mathrm{~h}$ half-life for BNT162b2 incubated platelets). At $24 \mathrm{~h}$ after intravenous ChAdOx1 nCov-19 administration, mouse platelets displayed an activated phenotype with increased P-Selectin (CD62P), compared to intramuscular injection (Fig. 1j).

Platelets are known to bind and shuttle blood-borne pathogens to professional phagocytes in the spleen and liver to mount an adaptive immune response [10-12]. In the spleen, platelets are abundant in the red pulp during steady state conditions, but deposit pathogens in the marginal zone and follicles, which are predominant regions of adaptive immune responses, upon pathogen binding [13]. We therefore analyzed platelet localization after i.v. compared to i.m. application in the spleen. To robustly label all platelets, we injected a fluorescent non-activating Gp1b-antibody at the time of vaccine injection. Analysis of overall Gp1b content in the spleen after i.v. injection in contrast to i.m. injection revealed prominent accumulation of $\mathrm{Gp} 1 \mathrm{~b}+$ material in the marginal zone and follicles; these areas are devoid of platelets under steady state conditions (Fig. 2a). In i.m injected animals, the Gp1b signal identified round and intact platelets in the splenic red pulp (Fig. 2a). In contrast, the Gp1b signal in the i.v. injection group appeared morphologically different with follicular and marginal zone clusters reminiscent of platelet remnants (Fig. 2a, Suppl. Fig. 1g). Professional phagocytes, particular red pulp macrophages, phagocytose activated or opsonized platelets in the spleen [14]. In line with this, we found increased platelet uptake by splenic F4/80 macrophages after ChAdOx1 nCov-19 i.v. compared to i.m. administration (Fig. 2b). In summary, these data show phagocytic uptake and processing of platelets and adenovirus in the spleen.

Even under steady-state conditions, contacts between passing platelets and local macrophages are frequently observed in the red pulp[15]. To understand the dynamics of platelet-adenovirus trafficking in more detail, we therefore co-transfused labeled control and ChAdOx1 nCov-19 incubated platelets and performed 4D intravital microscopy of the spleen (Suppl. Fig. 1h). We observed increased phagocytosis of

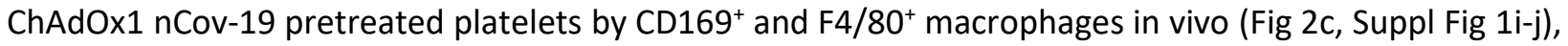
again pointing towards preferential targeting of virus-loaded platelets to professional phagocytes in the spleen. As a result, single-cell tracking in the spleen revealed decreased motility and enhanced adherence of the ChAdOx1 nCov-19 pretreated platelets to macrophages (Fig. $2 \mathrm{~d}$ and Suppl. Video 1 ).

Based on the platelet uptake by splenic macrophages, we hypothesized that presentation of platelet antigens in the spleen might trigger anti-platelet antibody production resulting in a second, delayed wave of platelet depletion occurring with a characteristic lag time[16]. This resembles the kinetics of clinically apparent thrombocytopenia of TTS patients reported to occur 4-30 days post vaccination. To test this hypothesis, we assessed adaptive immune responses in the spleen upon i.m./i.v. ChAdOx1 nCov-19 application. Intravenous ChAdOx1 nCov-19 led to significant splenomegaly with enlarged B cell follicles in the spleen (Fig. 2e-f). Next, we determined if this immune response results in production of antibodies targeting platelets. We incubated control mouse platelets with plasma from i.m./i.v. ChAdOx1 nCov-19 treated animals 6 days post injection and control plasma. Naïve ChAdOx1 nCov-19 unexperienced platelets incubated with plasma from intravenously injected mice showed increased IgM and IgG binding, pointing towards circulating anti-platelet antibodies (Fig. $2 \mathrm{~g}$ ). These did not lead to platelet activation, most likely because mouse platelets unlike their human counterparts lack Fc-receptors (Suppl. Fig 1k). As expected, plasma from ChAdOx1 nCov-19 intravenously injected antibody deficient $\mathrm{AlD}^{-/-} \mathrm{slgM}^{-/-}$showed a signal comparable to controls. We also screened the patient who presented with PF4/polyanion antibody negative thrombocytopenia and SVT for antiplatelet antibodies. We found anti-platelet 
antibodies in this patient that were reactive to the platelet receptor GPIIb/IIla, , although this result is limited by the fact that diagnostics were performed after IVIG treatment (Suppl Table 3).

Here, we use in vitro and murine models of ChAdOx1 nCov-19 vaccination to show that intravenous injection of this vaccine triggers platelet-adenovirus vector aggregate formation and platelet activation. This leads thrombocytopenia and uptake of these aggregates by professional phagocytes in the spleen. As a result, platelet and adenovirus antigens trigger the formation of platelet directed autoantibodies, as demonstrated by increased circulating anti-platelet IgG and IgM. Our findings might explain the rare incidence of TTS, as accidental intravenous deltoid injection is considered an unlikely event for anatomical reasons [17]. The CDC does not recommend aspiration during intramuscular application of vaccines [18]. However, in the case of adenovirus-based vaccines, aspiration prior to injection could offer a simple preventive measure against TTS.

So far, TTS has been linked exclusively to the presence of platelet-activating PF4-polyanion antibodies [3]. Here we report a patient with a TTS-like syndrome and no detectable PF4-polyanion antibodies similar to published reports $[7,8]$. Non-platelet-activating PF4/polyanion antibodies appear only in low frequencies in both adenovirus- and mRNA-vaccinated patients, making it unlikely that antibody formation against these targets is common after vaccination $[19,20]$.

It is important to note that we used an animal model which inherently cannot fully recapitulate disease pathophysiology observed in humans. Murine platelets do not express Fcy receptors, and therefore are not suited as a model of antibody mediated platelet activation and thrombocytopenia, i.e. upon the injection of heparin and PF4/polyanion antibody [21]. Similarly, we did not observe delayed antibodydependent thrombocytopenia after i.v. adenovirus injection in mice and did not detect platelet activation upon antibody binding.

Nevertheless, our data provide first experimental support for the potential sequence of events that could lead to TTS in some patients: Thrombocytopenia might be a result of accidental intravenous vaccine injection with ensuing platelet-adenovirus aggregation, which in turn triggers platelet activation and subsequent splenic clearance (Suppl. Fig. 3). This process is antibody independent and most likely clinically inapparent. Phagocytosis and processing of platelet-adenovirus aggregates by professional phagocytes then creates a cross-reactive immune response, which culminates in the delayed emergence of plateletbinding-antibodies. These platelet-binding-antibodies might induce a second wave of clinically apparent thrombocytopenia through platelet activation or opsonization. In fact, pro-thrombotic antibodies present in heparin induced thrombocytopenia Type II, and platelet opsonization with antibodies is a typical feature of immune thrombocytopenic purpura (ITP) Antibodies targeting platelets might shift these platelets towards a more thrombotic phenotype, which then leads to thrombosis in individuals with additional risk factors, including female sex, smoking or contraception. [22, 23]. Interestingly, we also detected platelet autoantibodies in the described patient case. Further research is needed to fully characterize the antibody responses in patients with TTS, as well as animal studies to define mechanisms and targets of the mounted adaptive immune response in depth. 


\section{Suppl. Methods section}

\section{Patient data}

The patient was treated at the university hospital LMU Munich. The patient consented to taking part in this study. The study was approved by local ethics committee of Munich. Data on presentation, treatment and outcome are depicted in Fig 1a-b, Suppl. Fig 1a and the laboratory values table (Suppl Table 1-3). A HIT-IL-Acustar assay and a HYPHEN BioMed HIT-ELISA Test deemed sensitive for TTS returned negative for this patient [5]. PF4 induced platelet activation(PIPA) and heparin induced platelet activation (HIPA) tests were performed as previously reported[24].

Suppl. Table 1

\begin{tabular}{|c|c|c|c|}
\hline \multicolumn{4}{|c|}{ Laboratory values at admission } \\
\hline \multicolumn{2}{|l|}{ Parameter } & Unit & Range \\
\hline INR & 1.1 & - & $0.8-1.2$ \\
\hline aPTT & 31 & $\mathrm{sec}$ & $22-34$ \\
\hline CRP & 0.2 & $\mathrm{mg} / \mathrm{dl}$ & $\leq 0.5$ \\
\hline D-Dimer & 1.6 & $\mu \mathrm{g} / \mathrm{ml}$ & $\leq 0.5$ \\
\hline Leukocytes & 8.68 & $\mathrm{G} / \mathrm{l}$ & $3.9-9.8$ \\
\hline Hemoglobin & 16.5 & $g / d l$ & 13.5-17.5 \\
\hline Platelets & 118 & $\mathrm{G} / \mathrm{I}$ & $146-328$ \\
\hline \multicolumn{4}{|l|}{ Thrombophilia screening } \\
\hline Factor-V-Leiden mutation & \multicolumn{3}{|c|}{$1691 \mathrm{G}>\mathrm{A}$ not detected } \\
\hline Prothrombin mutation & \multicolumn{3}{|c|}{ 20210G>A not detected } \\
\hline Lupus anticoagulant & \multicolumn{3}{|c|}{ not detected } \\
\hline APC screening & \multicolumn{3}{|c|}{4,4 (range $>=2,5$ ) } \\
\hline Protein C & \multicolumn{3}{|c|}{$78 \%$ (range 68-150) } \\
\hline Protein S & \multicolumn{3}{|c|}{ 91\% (range $74-136$ ) } \\
\hline
\end{tabular}

Suppl. Table 2

\begin{tabular}{|l|l|}
\hline \multicolumn{2}{|l|}{ HIT tests } \\
\hline Heparin/PF4 Ab Screening Test & positive \\
\hline HIT-IL-Acustar-Assay & negative \\
\hline HIT-ELISA-Test (HYPHEN Biomed) & negative \\
\hline HIPA test & negative \\
\hline PIPA test & negative \\
\hline
\end{tabular}

Suppl. Table 3

\begin{tabular}{|l|l|}
\hline \multicolumn{2}{|l|}{ Platelet auto-antibody panel } \\
\hline PakAuto $^{\circledR}$ Test* $^{*}$ & positive \\
\hline GPIb/IX Auto-antibodies* & negative \\
\hline
\end{tabular}




\begin{tabular}{|l|l|}
\hline GPla/Ila Auto-antibodies* & negative \\
\hline GPIIlb/IIla Auto-antibodies* & positive \\
\hline
\end{tabular}

*diagnostics performed after IVIG treatment initiation

\section{Mouse Strains}

C57BL/6 mice were purchased from The Jackson Laboratory. AID ${ }^{-/} \mathrm{slgM}^{-/-}$mice were bred and maintained at our animal facility. Mice were aged between 12-16 weeks for the experiments and were age and sexmatched for inter-group analyses. All mice live in standardized conditions where temperature, humidity, and hours of light and darkness are maintained at a constant level and provided water and food ad libitum. All animal experiments were performed in compliance with the relevant ethical regulations and were approved by the local administrative authority for the protection of animals (Regierung von Oberbayern, München).

\section{Vaccine acquisition}

Only residual content of discarded BNT162b2 (Pfizer-BioNTech) and ChAdOx1 nCov-19 (AstraZeneca) vials, that, according to the supervising physicians' body (Kassenärztliche Vereinigung Bayerns), could not be used for human application anymore, were used for this study. For ChAdOx1 nCov-19 this meant a storage period greater than $48 \mathrm{~h}$ after first withdrawal of a dose from a multi-dose vial, for BNT162b2 it meant a storage period greater than $24 \mathrm{~h}$ after first withdrawal of a dose from a multi-dose vial. Both vaccines were stored continuously at $4^{\circ} \mathrm{C}$. Experiments were conducted as soon as possible after vaccine acquisition.

\section{Human platelet isolation}

Human blood for in-vitro assays was obtained after informed consent from healthy male and female donors aged 20-35. Blood was collected with a syringe containing 1/7 of the blood volume citrate ( $39 \mathrm{mM}$ citric acid, $75 \mathrm{mM}$ sodium citrate, $135 \mathrm{mM}$ dextrose; $A C D)$. The blood was further diluted 1:1 with Tyrode's buffer Tyrode's buffer (137 mM NaCl, $2.8 \mathrm{mM} \mathrm{KCl}, 12 \mathrm{mM}$ NaHCO3, $5.5 \mathrm{mM}$ glucose, $10 \mathrm{mM}$ HEPES, pH = 6.5 ), centrifuged at $70 \mathrm{~g}$ for $35 \mathrm{~min}$, and the supernatant platelet-rich-plasma (PRP) was taken. PRP was further spun down after adding $0.1 \mathrm{mg} / \mathrm{ml} \mathrm{PGI} 2$ at $1200 \mathrm{~g}$ for $10 \mathrm{~min}$. The resulting washed platelet pellet was resuspended in $7.2 \mathrm{pH}$ Tyrode's buffer and used for subsequent experiments.

\section{Mouse platelet isolation}

Mouse blood was obtained via retro-orbital blood collection, after anesthesia with medetomidine, midazolam and fentanyl (MMF). Blood was collected via a capillary and mixed with $1 / 7$ of the blood volume citrate. The blood was further diluted 1:1 with Tyrode's buffer $(137 \mathrm{mM} \mathrm{NaCl}, 2.8 \mathrm{mM} \mathrm{KCl}, 12 \mathrm{mM}$ NaHCO3, $5.5 \mathrm{mM}$ glucose, $10 \mathrm{mM}$ HEPES, $\mathrm{pH}=6.5$ ), centrifuged at $70 \mathrm{~g}$ for $10 \mathrm{~min}$, and the supernatant platelet-rich-plasma (PRP) was taken. PRP was further spun at $1200 \mathrm{~g}$ for $10 \mathrm{~min}$. The resulting washed platelet pellet was resuspended in 7.2pH Tyrode's buffer and used for subsequent experiments.

\section{Platelet incubation with vaccines}

Ca. $1 \times 10^{8}$ washed platelets were incubated with $\sim 5 \times 10^{7}$ ChAdOx $1 \mathrm{nCov}-19$ viral particles $(100 \mu \mathrm{l})$, or $100 \mu \mathrm{l}$ BNT162b2 or PBS for 20min. After this 1:200 X649 (mouse, emfret Analytics) or CD41 (human, Biolegend) was added and further incubated for $10 \mathrm{~min}$ and then analyzed via flow-cytometry. For transfusion, after 
separate vaccine incubation with ChAdOx1 nCov-19 and BNT162b2, platelets were stained with 1:200 X649 or X488 (emfret Analytics) respectively for 10min. X488 and X649 labelling was switched for half of the mice to ensure no color bias. The platelets were spun down at $1200 \mathrm{~g}$ for $10 \mathrm{~min}$, resuspended in $200 \mu \mathrm{l}$ $7.2 \mathrm{pH}$ Tyrode's buffer and immediately injected separately via tail vein injection.

\section{Mouse vaccine injection and blood collection}

$5 \mu$ l (low dose) or $50 \mu$ l of ChAdOx1 nCov-19, BNT162b2, PBS, or heat inactivated ChAdOx1 nCov-19 were used for injections. Heat inactivation of ChAdOx1 nCov-19 was achieved by heating the vaccine to $99^{\circ} \mathrm{C}$ for $30 \mathrm{~min}$, then spinning the resulting protein precipitate down at $10,000 \mathrm{~g}$ for $3 \mathrm{~min}$ and taking the supernatant. Injection was performed either via tail vein or in the medial aspect of the thigh of isoflurane anesthetized mice. At specified time points, vaccine injected, and transfused mice were briefly anesthetized with isoflurane and blood was obtained via facial vein puncture and collected in EDTA capillaries. Blood counts were measured with the Sysmex XN-V Series XN-1000V cell counter, only blood counts that passed internal quality control were used.

\section{Platelet bound immunoglobulin detection}

Mouse washed platelets were incubated with 1:4 plasma of mice $6 \mathrm{~d}$ post-inoculation (p.i.) and control mice for 40min. After adding 1:100 CD41 PB, IgG Cy3 and IgM FITC and 20min incubation washed platelets were fixed, diluted and analyzed via flow cytometry.

\section{Intravital imaging of the spleen}

Co-injection of platelets was performed as previously described[25]. After injection of X488 or X649 labelled platelets incubated with ChAdOx1 nCov-19 or PBS, mice were also injected with $15 \mu$ l each of CD169 PE and F4/80 BV421 (Biolegend), anesthetized with MMF, shaven, and the spleen exposed via a small lateral cut. Subsequently the spleen was mobilized and imaged with a Zeiss LSM 880 confocal microscope (x20 obj.) in airyscan mode. Regular blood flow in the spleen was observed throughout imaging, and z-Stack images ( $1 \mu \mathrm{m}$ slice thickness) as well as videos were taken.

\section{Flow cytometry}

Flow cytometry to characterize platelet profiles was performed as previously described [26]. Briefly, incubation with panels at 1:200 per antibody of either washed platelets or whole blood, at least $5 x$ the amount of FACS Lysing Solution (BD) was added to fixate platelets and lyse blood. Flow cytometry was performed on a BD LSRFortessa and BD FACSCanto. Flowjo (BD) was used for flowcytometric analysis, gating strategies are shown in Supplemental Figure 2. For adenovirus binding, as well as IgM and IgG binding a positive gate was used, for MFIs the MFI of each marker per data point was normalized by dividing it by the highest MFI of that marker and experimental run.

Antibodies used for Flow Cytometry:

\begin{tabular}{|c|c|c|c|}
\hline Color & Target & Species reactivity & Catalogue \# and company \\
\hline AF700 & CD44 & Mouse & $\# 103026$, biolegend \\
\hline APC & GPIb & Mouse & X649, emfret Analytics \\
\hline APC/Fire 750 & CD31 & Mouse & $\# 102433$, biolegend \\
\hline
\end{tabular}


bioRxiv preprint doi: https://doi.org/10.1101/2021.06.29.450356; this version posted June 29, 2021. The copyright holder for this preprint (which was not certified by peer review) is the author/funder, who has granted bioRxiv a license to display the preprint in perpetuity. It is made available under aCC-BY-NC-ND 4.0 International license.

\begin{tabular}{|c|c|c|c|}
\hline BV421 & CD41 & Mouse & \#133932, biolegend \\
\hline BV510 & AnV & Mouse & \#640937, biolegend \\
\hline BV711 & CD107a & Mouse & \#121631, biolegend \\
\hline PE-Cy7 & CD62p & Mouse & \#148309, biolegend \\
\hline PE-Dazzle & CD9 & Mouse & \#124821, biolegend \\
\hline PerCP-Cy5.5 & CD154 & Mouse & \#106513, biolegend \\
\hline Су3 & $\lg G$ & Mouse & \#M30010, ThermoFisher \\
\hline PE & GP2b3a & Mouse & M023-2, emfret Analytics \\
\hline FITC & $\operatorname{lgM}$ & Mouse & A21042, life technologies \\
\hline FITC & GPIb & Mouse & X488, emfret Analytics \\
\hline FITC & Hexon & Adenovirus & ab87333, abcam \\
\hline BV650 & CD63 & Human & \#353026, biolegend \\
\hline FITC & CLEC-2 & Human & \#372007, biolegend \\
\hline PE & CD162/PSGL1 & Human & \#328805, biolegend \\
\hline PERCP-Сy5.5 & CD36 & Human & \#336224, biolegend \\
\hline AF 647 & PAC-1 & Human & \#362806, biolegend \\
\hline APC-Cy7 & CD184 (CXCR4) & Human & \#306528, biolegend \\
\hline BV510 & CD42b & Human & \#303933, biolegend \\
\hline BV 711 & CD154 (CD40L) & Human & \#310837, biolegend \\
\hline AF 700 & CD41 & Human & \#133926, biolegend \\
\hline BV421 & CD62P & Human & \#304926, biolegend \\
\hline PE-Dazzle & CD31 (PECAM) & Human & \#303130, biolegend \\
\hline PE-Cy7 & CD284 (TLR4) & Human & \#312805, biolegend \\
\hline BV650 & CD63 & Human & \#353026, biolegend \\
\hline
\end{tabular}

\section{Histology}

Organs were harvested directly after sacrifice, fixed at 4\%PFA for $1 \mathrm{~h}$ and subsequently $30 \%$ sucrose overnight, then embedded in OTC and stored at $-80^{\circ} \mathrm{C}$. Sections were stained with antibodies against F4/80 (Biolegend, Cat. No. 123110), CD45R/B220 (Biolegend, Cat. No. 103212), ki67 (Abcam, Cat. No. ab15580), Lectin PNA (Thermofisher, Cat. No. L32458). Secondary antibodies and nucleic acid stain 
included FITC goat anti mouse (Thermofisher, Cat. No. A11029) and Cy3 goat anti rabbit (Jackson Immunoresearch, Cat. No. 111-165-003) and Hoechst33342. Micrographs were taken on a Zeiss LSM 880 confocal microscope in airyscan mode and a Leica LAS X epifluorescence microscope.

\section{Data analysis and statistics}

Analysis of histology and intravital imaging was done using ImageJ v2.1 or Imaris (Bitplane). Intravital imaging cell tracking was done manually on randomly selected cells which could be traced for at least 5 frames. Statistics were computed with Imaris, Meandering Index was defined as Net displacement/Total track length. Unpaired t-tests with Welch's correction was used for all cell tracking analyses. For area quantification, thresholds were taken with ImageJ and overlap quantified by percent area overlapping between thresholds. For all statistical tests Prism (GraphPad) was used. Unpaired t-test were used unless otherwise noted. All data are shown as mean \pm standard error of the mean (s.e.m.). When more than three t-tests were applied on the same data set, multiple testing correction was done.

\section{Figure Legends}

Figure 1 | Intravenous but not intramuscular injection of ChAdOx1 nCov-19 triggers ChAdOx1 nCov-19platelet binding and thrombocytopenia. a, Clinical time course of TTS patient, starting at vaccination with ChAdOx1-S (day 0). b, Coronal contrast enhanced computed tomography image of showing a likely sinus vein thrombosis (enlarged on the upper right). c, Exemplary flowcytometric contour plots of human washed-platelets with ChAdOx1 nCov-19, BNT162b2, or PBS. 2\% contour with outliers shown gate shows adenovirus positive platelets. d, Quantification of adenovirus platelet binding according to c. One-way ANOVA with post-hoc Tukey's test. Comparison of ChAdOx1 nCov-19 to both controls. $n \geq 3$ human donors per group. e, Platelet expression of activated GPIIbllla incubated with ChAdOx1 nCov-19 or BNT162b2. Normalized MFIs. Unpaired t-test. $n=8$ donors per group. Exemplary histogram of activated GPIIbllla is also shown. $\mathbf{f}$, Platelet counts of mice over time. Multiple t-tests with Holm-Sidak correction of ChAdOx1 nCov-19 i.v. and i.m. is shown. $n \geq 3$ per time point for of ChAdOx1 nCov-19 groups, $n \geq 2$ per time point for other groups. $\mathrm{g}$, Linear regression of platelet count at $24 \mathrm{~h}$ p.i. and adenovirus binding to platelets $1 \mathrm{~h}$ p.i.. $95 \%$ confidence interval shown, $p$ value denotes line deviation from zero. $\mathbf{h}$, Adenovirus-platelet binding after i.m. or i.v. administration of ChAdOx1 nCov-19 $1 \mathrm{~h}$ and $24 \mathrm{~h}$ post inoculation. Unpaired t-tests. $\mathrm{n} \geq 4$ per group. i, Ratio of transfused BNT162b2 and ChAdOx1 nCov-19 platelets (total transfused platelets are normalized to $100 \%$ ) over time. Unpaired t-tests. $n=4$ per time point. j, Platelet surface marker expression of mouse platelets $24 \mathrm{~h}$ after administration of ChAdOx1 nCov-19 i.v. or i.m.. Normalized MFIs. Multiple ttests with Holm-Sidak correction. $\mathrm{n}=7$ mice per group. Error bars are mean \pm s.e.m. ${ }^{*} \mathrm{p}<0.05,{ }^{* *} \mathrm{p}<0.01$, $* * * p<0.001$.

Figure 2 | Platelet-adenovirus aggregates are taken up by macrophages in the spleen and lead to adenovirus-platelet directed immune responses. a, Images of X649 endogenous platelet labelling mice 24h after i.m. and i.v. ChAdOx1 nCov-19 administration. Cut out of red pulp to follicle transition is shown on the upper left. Scale bars for overview are $50 \mu \mathrm{m}$, for cut outs $10 \mu \mathrm{m}$. Quantification of Platelet (X649) area in the marginal and follicle zone as percent of marginal and follicular area. $n=4$ per group, unpaired t-test. b, Quantification of platelet-F4/80 co-localization in the splenic red pulp. Co-localization is shown as percent of total platelet- $\mathrm{F} 4 / 80$ area. $\mathrm{n}=4$ per group, unpaired t-test. $\mathrm{c}, 3 \mathrm{D}$ reconstruction of in-vivo microscopy of the spleen with transfused platelets pretreated with either ChAdOx1 nCov-19 (white) or 
PBS (green). ChAdOx1 nCov-19 pretreated platelets are taken up by $\mathrm{F} 4 / 80^{+} / \mathrm{CD} 169^{+}$macrophages, while control treated platelets are not. Scale bar is $5 \mu \mathrm{m}$. d, Platelet tracking parameters of intraviral imaging. $\mathrm{n}=31$ platelets tracked per group of one mouse. 10 exemplary tracked paths per group are shown on the left as $\mathrm{x}$ - and $\mathrm{y}$-displacement, ticks signify $5 \mu \mathrm{m}$ intervals. Unpaired t-test with Welch's correction. e, Spleen weights of animals $6 \mathrm{~d}$ p.i.. Representative images of spleens of both groups are shown, scale bar is $2 \mathrm{~cm}$. Unpaired t-test. $\mathrm{n}=5$ per group. $\mathrm{f}$, Quantification of $\mathrm{B} 220^{+}$are in the spleen of i.v. or i.m. ChAdOx1 nCov19 injected mice $6 \mathrm{~d}$ p.i. as percentage of total splenic area. Unpaired t-test, $n=3$ per group. Representative images of a splenic micrograph of both groups are shown, scale bar is $100 \mu \mathrm{m}$. $\mathbf{g}$, Control mouse platelets positive for bound IgM and IgG after incubation with plasma from mice $6 \mathrm{~d}$ p.i. with either i.m. or i.v. ChAdOx1 nCov-19 or ChAdOx1 nCov-19 AID $/-$ slgM ${ }^{-/}$mice for IgG additionally. $\mathrm{n}=5$ per group for i.v. or i.m., $\mathrm{n}=4$ for $\mathrm{AlD}^{-/} \mathrm{slgM}^{-/}$. Unpaired t-tests between i.v. ChAdOx1 nCov-19 and the other groups. Percent of platelets binding IgM or IgG are normalized to the mean of 4 control plasmas. Error bars are mean \pm s.e.m. ${ }^{*} \mathrm{p}<0.05, * * \mathrm{p}<0.01, * * * \mathrm{p}<0.001$.

Supplementary Figure 1 | a, Time course of hemoglobin, leukocyte and platelet counts of SVT patient. b, Platelet surface marker expression of human platelets incubated with ChAdOx1 nCov-19 or BNT162b2. Normalized MFIs. Multiple t-tests with Holm-Sidak correction, non-significant. $n=8$ donors per group. $c$, Quantification of adenovirus platelet binding to mouse platelets. One-way ANOVA with post-hoc Tukey's test. Comparison of ChAdOx1 nCov-19 to both controls. $n=4$ per group. $d$, Cell count parameters of blood taken at 24h p.i. with either ChAdOx1 nCov-19 i.v. or i.m., BNT162b2 i.v. or PBS i.m.. Two-way ANOVA with post-hoc Tukey's test, all nonsignificant. $n \geq 5$ per group. e, Comparison of platelet count at $24 h$ p.i. with either ChAdOx1 nCov-19 i.v. or i.m. (same data as in Figure 1f) or low-dose ChAdOx1 nCov-19 i.v. administration. Unpaired t-tests, $\mathrm{n} \geq 4$ per group. $\mathrm{f}$, Time course of platelet counts right before and after ChAdOx1 nCov-19 i.v. administration of $\mathrm{AlD}^{-/} \mathrm{slgM}^{-/-}$mice. Unpaired t-tests, $\mathrm{n}=4$ per time point. g, Zoomin of the crop outs in Figure $2 \mathrm{a}$. Scale bars are $5 \mu \mathrm{m}$. h, Illustration of intravital splenic imaging with transfused platelets and representative overview image of intravital microscopy. Scale bar $10 \mu \mathrm{m}$. i, Zprojection of a 3D-stack intravital splenic microscopic image showing phagocytosed ChAdOx1 nCov-19 pretreated transfused platelets (white, arrow) in comparison to PBS treated platelets (green). Scale bar $5 \mu \mathrm{m}$. j, Series of images from an intravital splenic microscopic video showing phagocytosis (upper arrow) and interactions (lower arrow) of ChAdOx1 nCov-19 pretreated transfused platelets. Time is shown on the upper left, scale bars $5 \mu \mathrm{m}$. k, Platelet activation marker expression after plasma incubation of mice with i.v. or i.m. ChAdOx1 nCov-19 administration. Normalized MFIs. Unpaired t-tests, non-significant. $\mathrm{n}=4$ mice per group. Error bars are mean \pm s.e.m. ${ }^{*} p<0.05,{ }^{* *} p<0.01,{ }^{* * *} p<0.001$.

Supplementary Figure 2 | a, Gating strategy for human in-vitro platelet-adenovirus binding. Last gate was used to quantify platelet-adenovirus binding. b, Gating strategy for murine in-vitro platelet-adenovirus binding. Last gate was used to quantify platelet-adenovirus binding. c, Gating strategy for murine in-vivo platelet-adenovirus binding and platelet surface marker expression. Second gate was used to derive MFIs for platelet surface marker expression, last gate was used to quantify platelet-adenovirus binding. d, Gating strategy for transfused platelet tracking. Both of the last gates were used to quantify ChAdOx1 nCov-19 and BNT162b2 incubated transfused platelet fraction. e, Gating strategy for immunoglobulin binding of platelets incubated with plasma from vaccinated mice. Last gate was used to quantify either $\operatorname{lgG}$ (shown) or IgM binding. All gating is shown as contour plots with $2 \%$ counter and outliers shown.

Supplementary Figure 3 | Graphical abstract: Thrombocytopenia and splenic platelet directed immune responses after intravenous ChAdOx1 nCov-19 administration. Intramuscular injection of ChAdOx1 
bioRxiv preprint doi: https://doi org/10.1101/2021.06.29.450356; this version posted June 29, 2021. The copyright holder for this preprint (which was not certified by peer review) is the author/funder, who has granted bioRxiv a license to display the preprint in perpetuity. It is made available under aCC-BY-NC-ND 4.0 International license.

nCov-19 leads to normal vaccine response without platelet involvement. Accidental intravascular injection of ChAdOx1 nCov-19 leads to adenovirus-platelet binding and platelet activation. Platelets are cleared by professional phagocytes, particularly in the spleen. Trafficking and processing of platelet adenovirusaggregates from the red pulp to splenic follicles leads to a B cell response with the emergence of platelet binding antibodies. 


\section{REFERENCES}

1. Teijaro, J.R. and D.L. Farber, COVID-19 vaccines: modes of immune activation and future challenges. Nature Reviews Immunology, 2021: p. 1-3.

2. See, I., et al., US Case Reports of Cerebral Venous Sinus Thrombosis With Thrombocytopenia After Ad26. COV2. S Vaccination, March 2 to April 21, 2021. Jama, 2021.

3. Greinacher, A., et al., Thrombotic thrombocytopenia after ChAdOx1 nCov-19 vaccination. New England Journal of Medicine, 2021.

4. Franchini, M., G.M. Liumbruno, and M. Pezzo, COVID-19 Vaccine-associated Immune Thrombosis and Thrombocytopenia (VITT): diagnostic and therapeutic recommendations for a new syndrome. European Journal of Haematology, 2021.

5. Nazy, I., et al., Recommendations for the clinical and laboratory diagnosis of vaccine-induced immune thrombotic thrombocytopenia (VITT) for SARS-CoV-2 infections: Communication from the ISTH SSC Subcommittee on Platelet Immunology. 2021, Wiley Online Library.

6. Douxfils, J., et al., Hypotheses behind the very rare cases of thrombosis with thrombocytopenia syndrome after SARS-CoV-2 vaccination. Thrombosis Research, 2021. 203: p. 163-171.

7. Pottegård, A., et al., Arterial events, venous thromboembolism, thrombocytopenia, and bleeding after vaccination with Oxford-AstraZeneca ChAdOx1-S in Denmark and Norway: population based cohort study. bmj, 2021. 373.

8. Vayne, C., et al., PF4 Immunoassays in Vaccine-Induced Thrombotic Thrombocytopenia. NEJM, 2021.

9. Irvine, D.J., A. Aung, and M. Silva, Controlling timing and location in vaccines. Advanced Drug Delivery Reviews, 2020.

10. Stone, D., et al., Adenovirus-platelet interaction in blood causes virus sequestration to the reticuloendothelial system of the liver. Journal of virology, 2007. 81(9): p. 4866-4871.

11. Othman, M., et al., Adenovirus-induced thrombocytopenia: the role of von Willebrand factor and P-selectin in mediating accelerated platelet clearance. Blood, 2007. 109(7): p. 2832-2839.

12. Verschoor, A., et al., A platelet-mediated system for shuttling blood-borne bacteria to CD8alpha+ dendritic cells depends on glycoprotein GPIb and complement C3. Nat Immunol, 2011. 12(12): p. 1194-201.

13. Broadley, S.P., et al., Dual-track clearance of circulating bacteria balances rapid restoration of blood sterility with induction of adaptive immunity. Cell host \& microbe, 2016. 20(1): p. 36-48.

14. Norris, P.A.A., et al., FcyRI and FcyRIII on splenic macrophages mediate phagocytosis of antiglycoprotein Ilb/IIla autoantibody-opsonized platelets in immune thrombocytopenia. haematologica, 2021. 106(1).

15. Prislovsky, A. and T.S. Strom, Increased uptake by splenic red pulp macrophages contributes to rapid platelet turnover in WASP(-) mice. Experimental hematology, 2013. 41(9): p. 789-798.

16. Nutt, S.L., et al., The generation of antibody-secreting plasma cells. Nature Reviews Immunology, 2015. 15(3): p. 160-171.

17. Davidson, K.M. and L. Rourke, Teaching best-evidence: Deltoid intramuscular injection technique. Journal of Nursing Education and Practice, 2013. 3(7): p. 120.

18. ACIP, Vaccine Recommendations and Guidelines of the ACIP. Centers for Disease control and Prevention.

19. Thiele, T., et al., Frequency of positive anti-PF4/polyanion antibody tests after COVID-19 vaccination with ChAdOx1 nCoV-19 and BNT162b2. Blood, 2021.

20. Sørvoll, I.H., et al., An observational study to identify the prevalence of thrombocytopenia and anti-PF4/polyanion antibodies in Norwegian health care workers after COVID-19 vaccination. Journal of Thrombosis and Haemostasis, 2021. 
21. Reilly, M.P., et al., Heparin-induced thrombocytopenia/thrombosis in a transgenic mouse model requires human platelet factor 4 and platelet activation through FcyRIIA. Blood, 2001. 98(8): p. 2442-2447.

22. Arepally, G.M., Heparin-induced thrombocytopenia. Blood, The Journal of the American Society of Hematology, 2017. 129(21): p. 2864-2872.

23. Kuwana, M., Y. Okazaki, and Y. Ikeda, Splenic macrophages maintain the anti-platelet autoimmune response via uptake of opsonized platelets in patients with immune thrombocytopenic purpura. J Thromb Haemost, 2009. 7(2): p. 322-9.

24. Handtke, S., et al., A Flow cytometric assay to detect platelet activating antibodies in VITT after ChAdOx1 nCov-19 vaccination. Blood, 2021.

25. Nicolai, L., et al., Vascular surveillance by haptotactic blood platelets in inflammation and infection. Nature Communications, 2020. 11(1): p. 5778.

26. Nicolai, L., et al., Immunothrombotic dysregulation in COVID-19 pneumonia is associated with respiratory failure and coagulopathy. Circulation, 2020.

Acknowledgements:

Author contributions: $L N$ and AL conceived of and initiated the study. $L N$ conceptualized the study. LN, $A L, K P, K S$ and $S M$ planned the experiments. $L N, A L, K P, A A, E R, M H, V P, L E, D R$ and $A T$ conducted the experiments. $A L, L N, K P, A A, R E$ and $R K$ analyzed the results, $A L$ visualized the findings. $L N$ wrote the initial draft, $L N, A L, K P, K S$ and $M S$ edited the manuscript with input from all authors. KaS and MI provided resources. LN, KP, KS and SM provided oversight, resources and funding.

We thank all lab members for their valuable input and help, as well as Dr. Schneider for his substantial support.

Competing interests: The authors declare no conflict of interest.

Materials \& correspondence: Materials are shared upon reasonable request by email.

Funding: This study was supported by the Deutsche Herzstiftung e.V., Frankfurt a.M. [LN], Deutsche Forschungsgemeinschaft (DFG) SFB 914 (S.M. [B02 and Z01], K.S. [B02]), the DFG SFB 1123 (S.M. [B06], K.S. [A07]), the DFG FOR 2033 (S.M.), the German Centre for Cardiovascular Research (DZHK) (Clinician Scientist Programme [L.N.], MHA 1.4VD [S.M], FP7 program (project 260309, PRESTIGE [S.M.]), and the DFG Clinician Scientist Programme PRIME (413635475, K.P., R.K.). The work was also supported by the European Research Council (ERC-2018-ADG “IMMUNOTHROMBOSIS" [S.M.] and ERC- "T-MEMORE" [K.S.])

Corresponding authors: leo.nicolai@med.uni-muenchen.de, alexander.leunig@med.uni-muenchen.de, kami.pekayvaz@med.uni-muenchen.de \& Konstantin.stark@med.uni-muenchen.de, Medizinische Klinik und Poliklinik I, University Hospital Ludwig-Maximilian-University Munich, Marchioninistr. 15 81377, Munich, Germany. 
Figure 1 | Intravenous but not intramuscular injection of ChAdOx1 nCov-19 triggers ChAdOx1 nCov-19-platelet binding and thrombocytopenia.

a

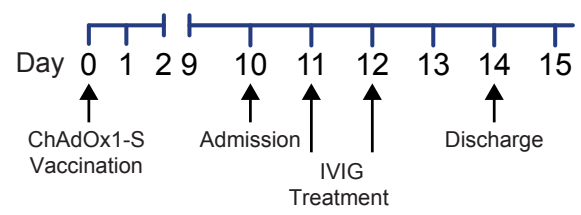

b

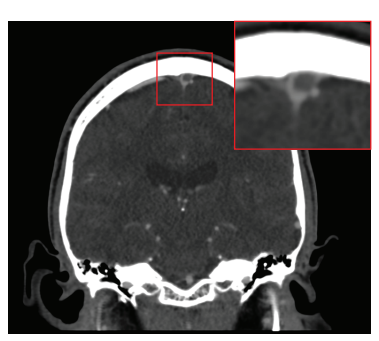

e

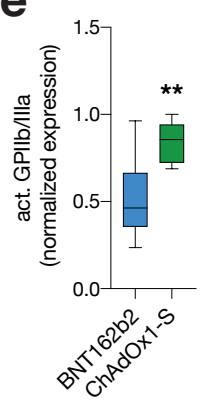

i

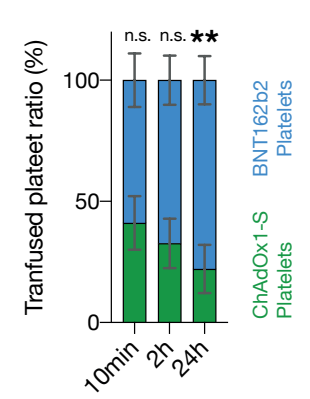

f

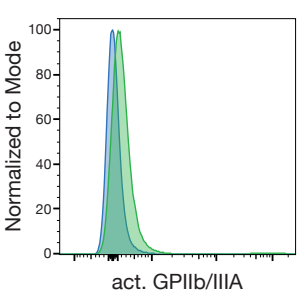

j

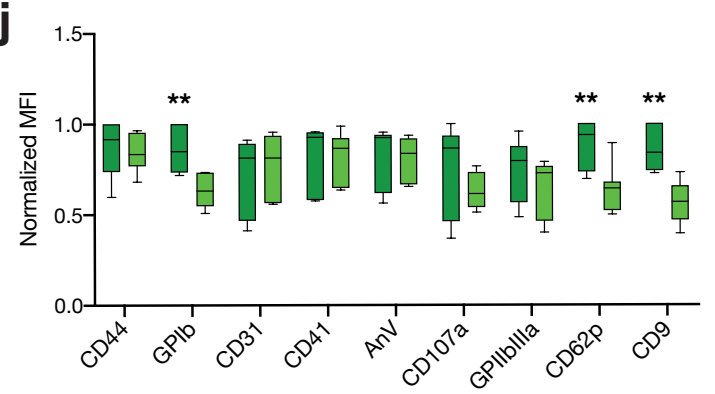

C
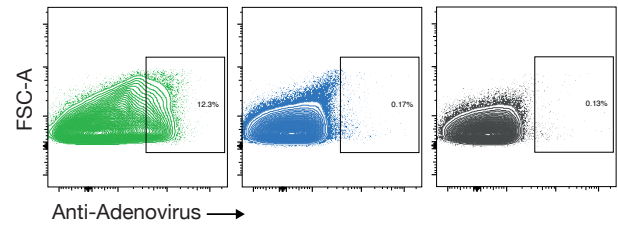

ChAdOx1-S iv BNT162b2 iv

PBS

g

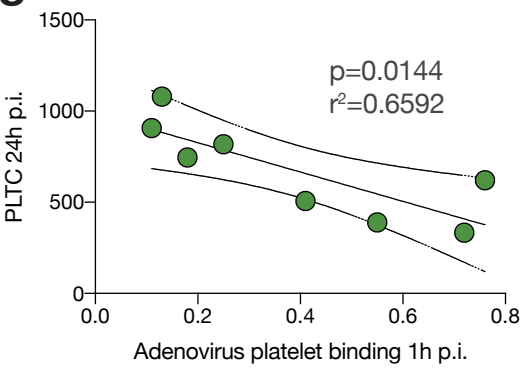

ChAdOx1-S iv

ChAdOx1-S im

heat inactivated $\mathrm{ChAdO} \times 1-\mathrm{S}$

BNT162b2 iv

PBS h

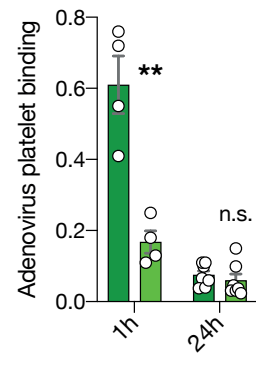

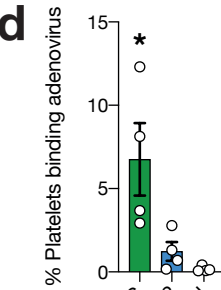
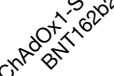
Figure 2 | Platelet-adenovirus aggregates are taken up by macrophages in the spleen and lead to adenovirus-platelet directed immune responses..

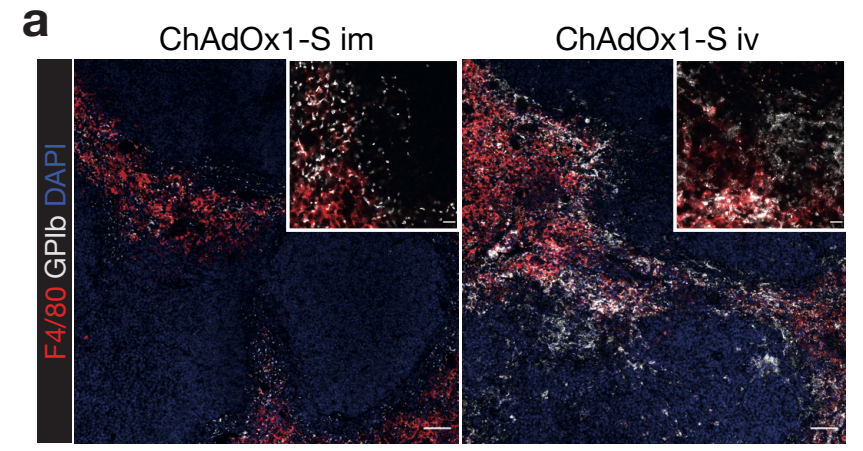

d
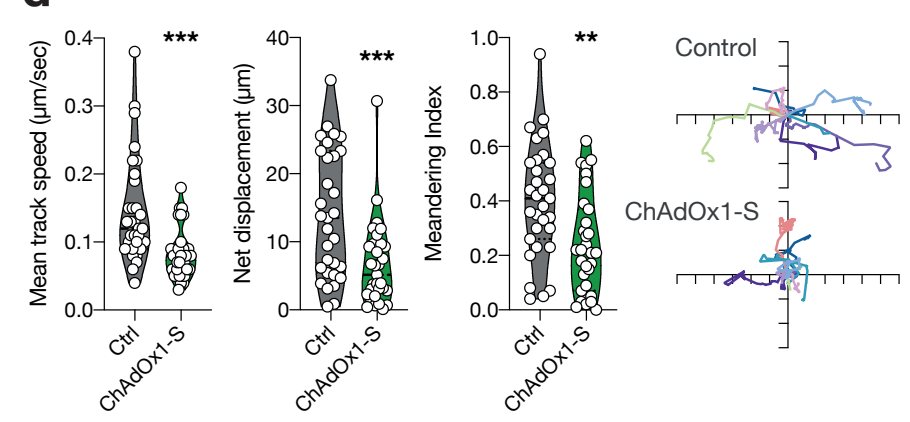

g
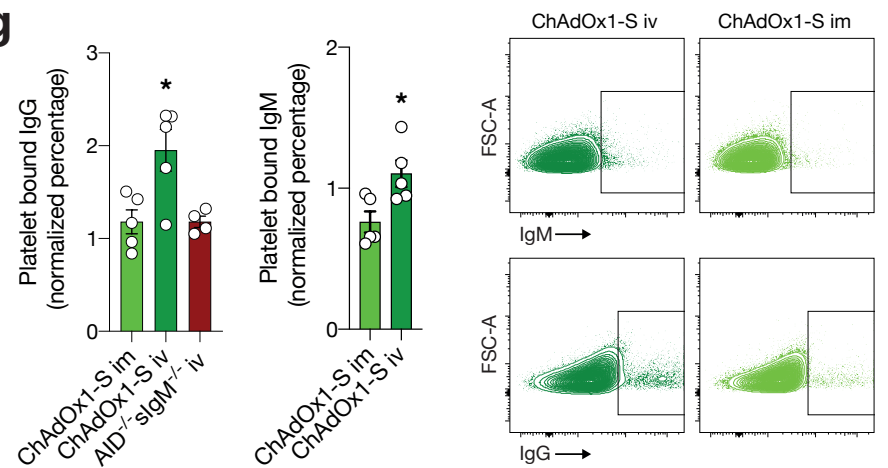

b
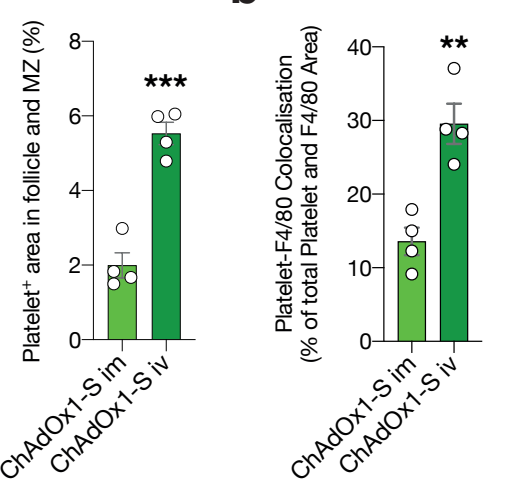

e

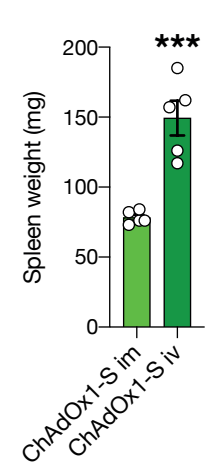

C

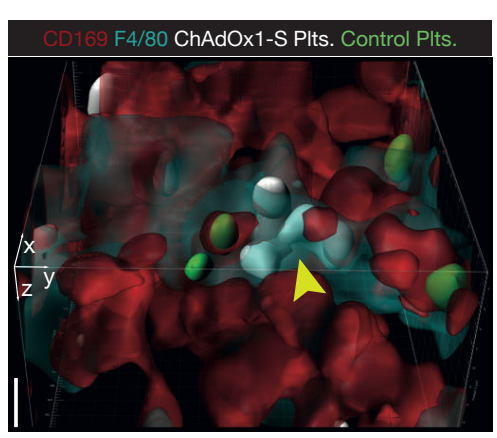

f

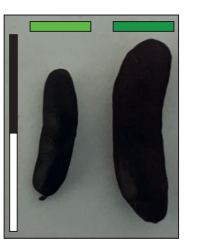

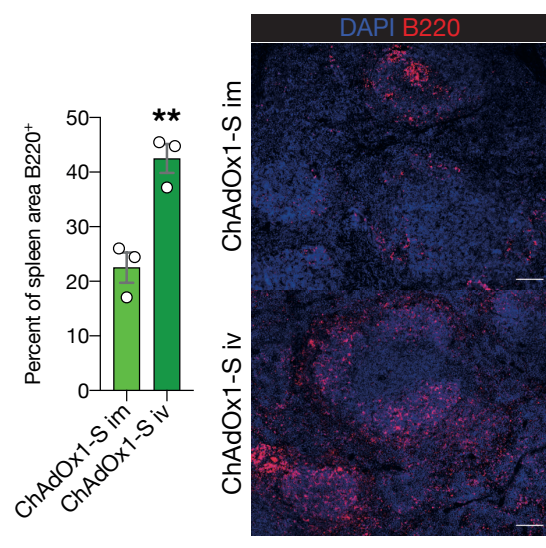


a

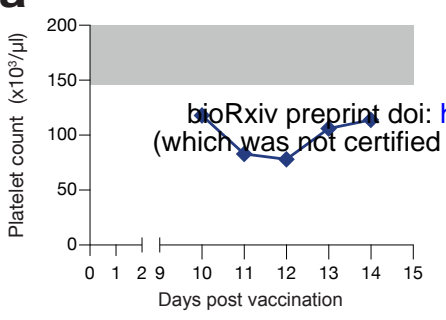

b

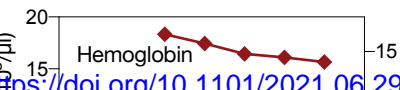

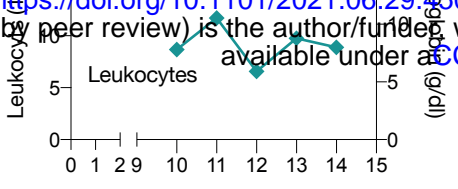

d

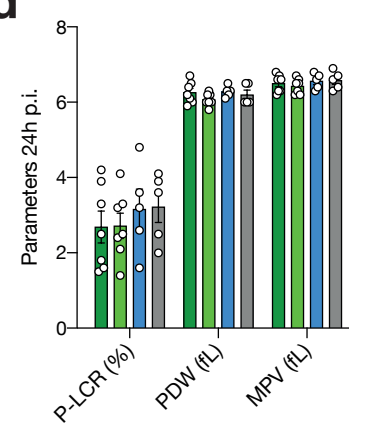

h

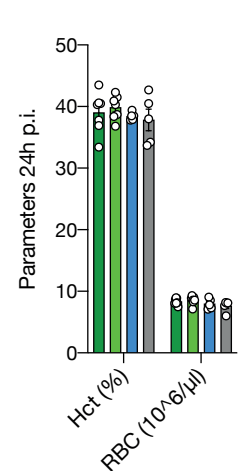

e
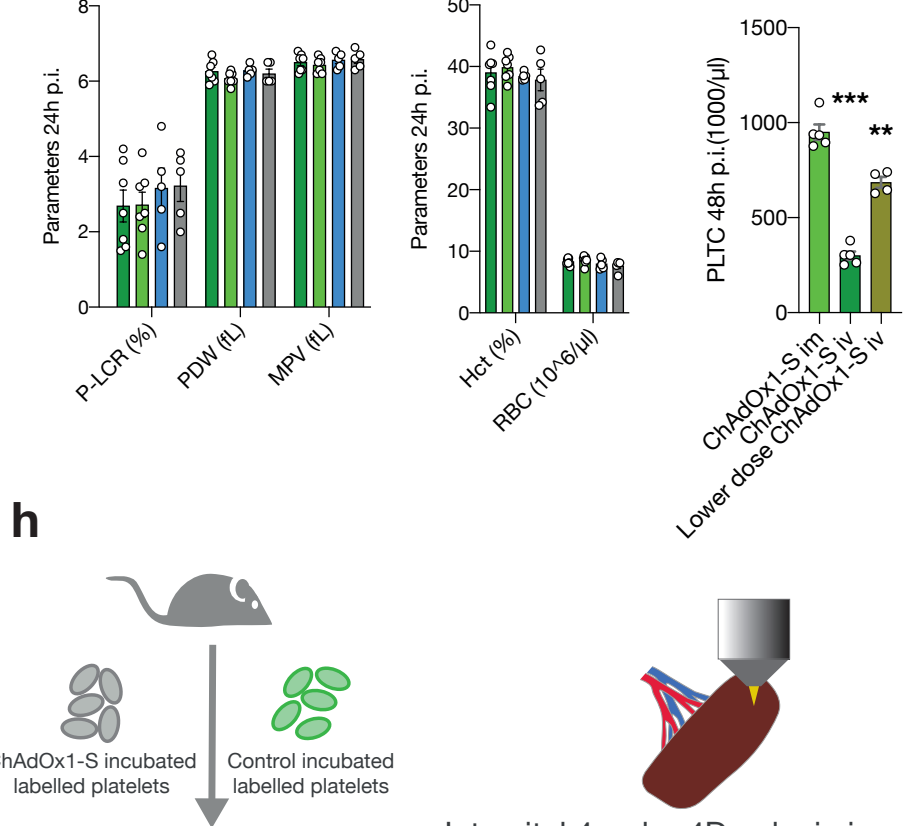

Transfusion

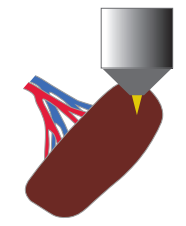

Intravital 4-color 4D splenic imaging with $F 4 / 80$ and CD169 labelling

f

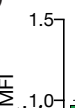

${ }_{1.0}^{1.5}$
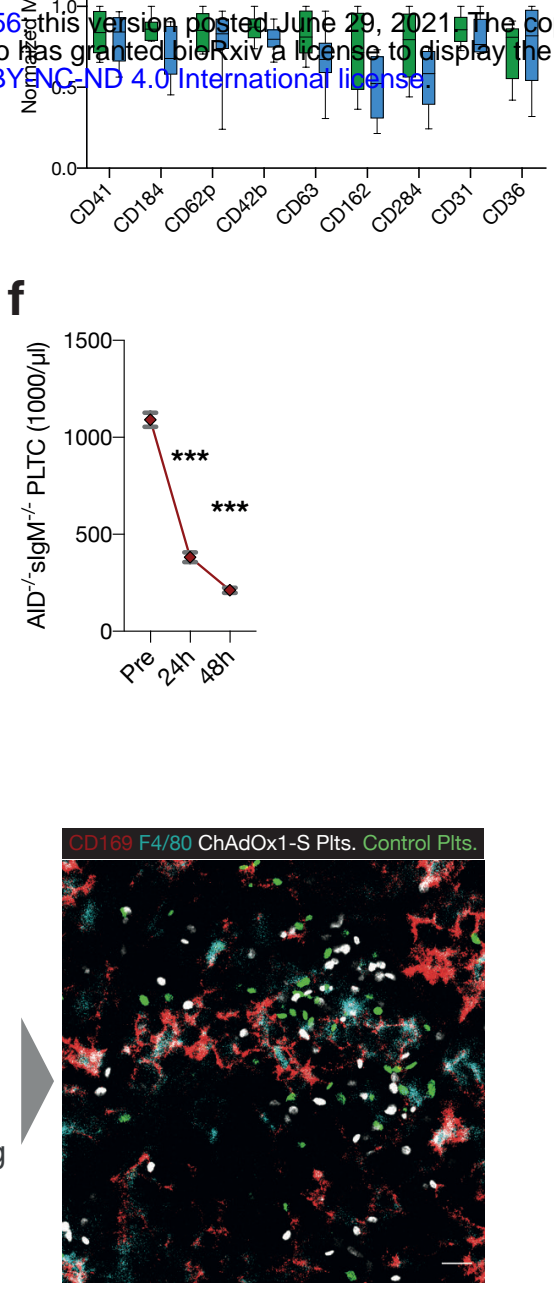

j

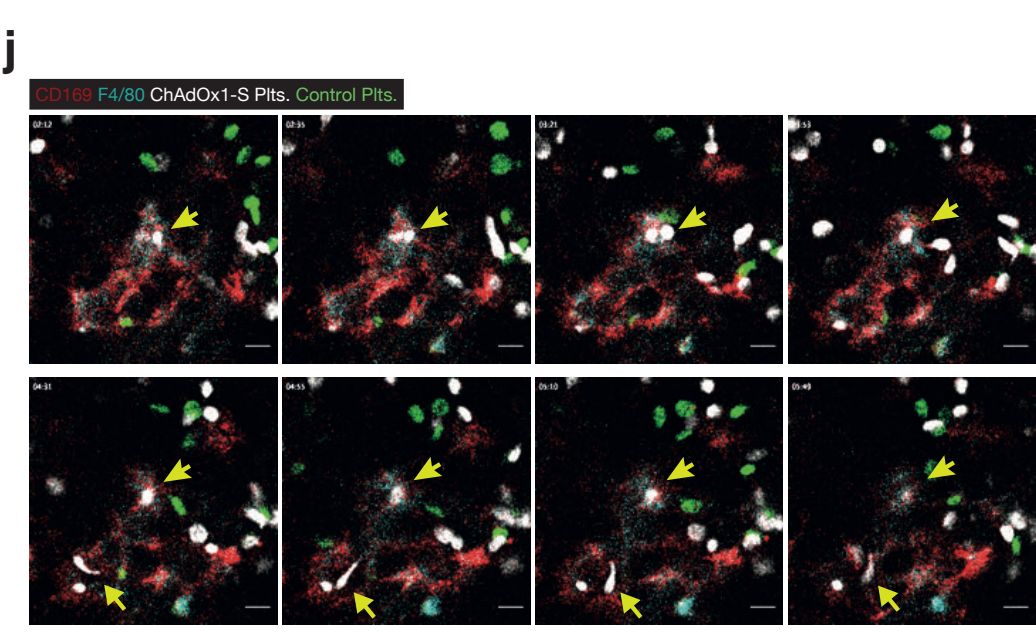

C ${ }^{2}{ }^{\frac{0}{2}}{ }^{6}{ }^{\text {Mouse }}$

ht Iolder for this preprint

in in perpetuity. It is made ChAdOx1-S iv $^{-\frac{10}{6}}$

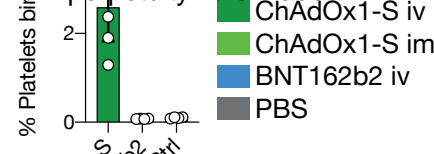

BNT162b2 iv $x^{5} x^{2} c^{x+1}$

cor

$E$
0
1
$\frac{1}{x}$
0
$\frac{0}{4}$
0

g
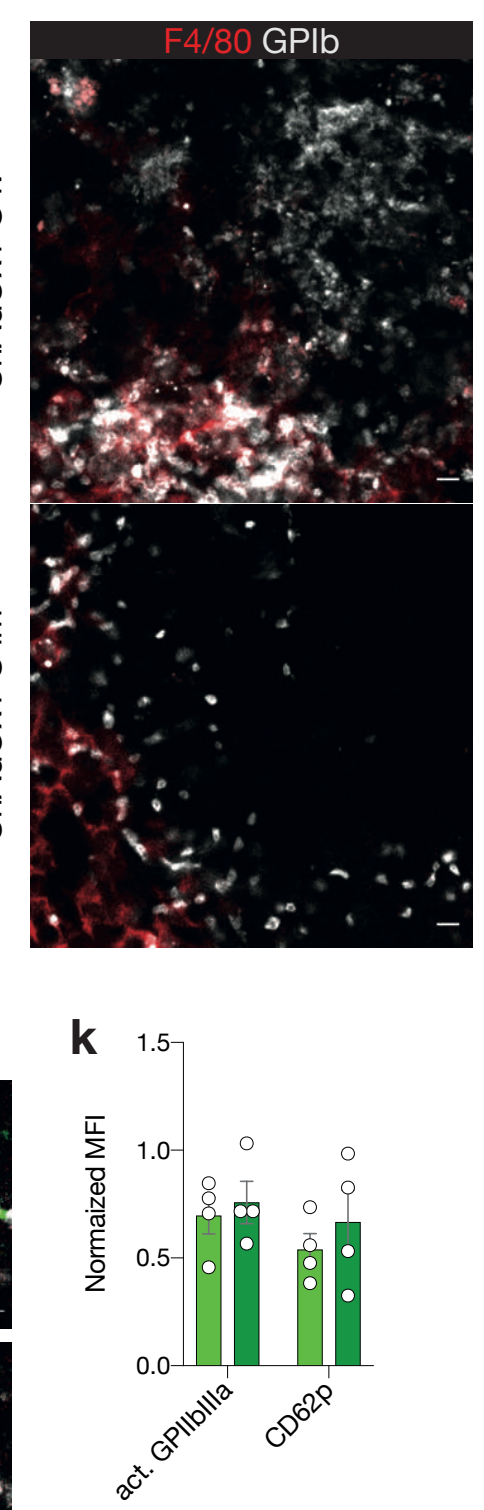

i

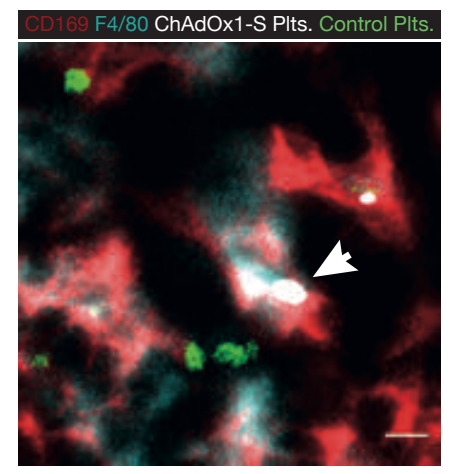


a

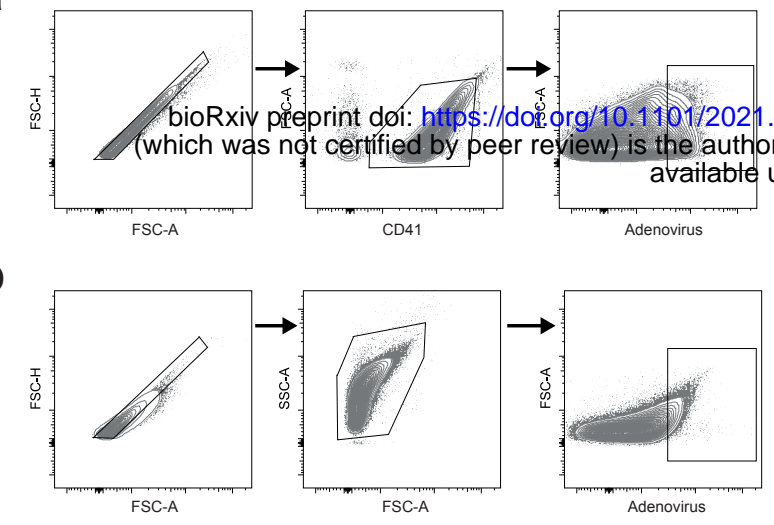

C

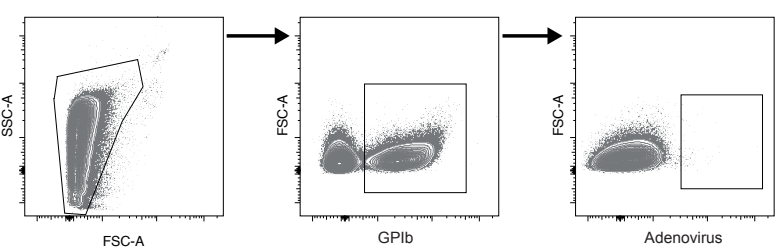

d

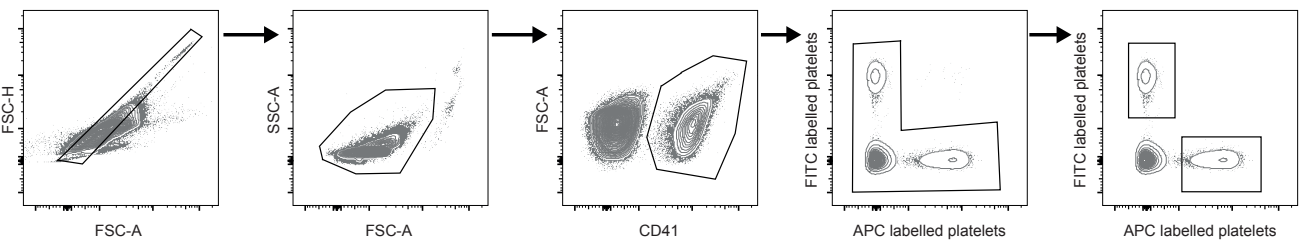

e

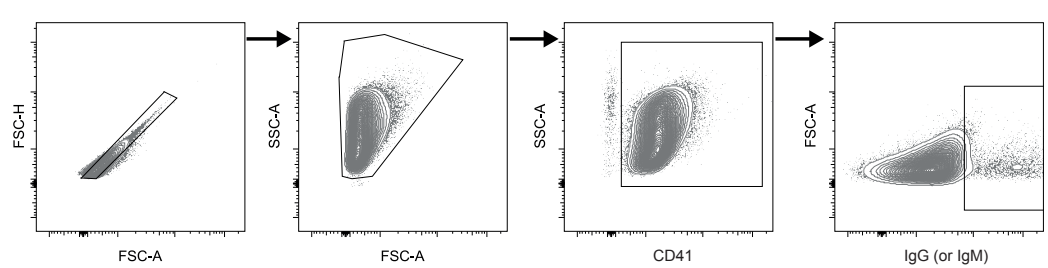


